\title{
OPEN Diagnostic performance of cone-beam computed tomography for scaphoid fractures: a systematic review and diagnostic meta-analysis
}

Ta-WeiYang ${ }^{1,2}$, Yen-Yue Lin ${ }^{1,2}$, Shih-Chang Hsu ${ }^{3,4}$, Karen Chia-Wen Chu ${ }^{3,4}$, Chih-Wei Hsiao ${ }^{2,5}$, Chin-Wang Hsu ${ }^{4,5}$, Chyi-Huey Bai ${ }^{6}$, Cheng-Kuang Chang ${ }^{7}$ \& Yuan-Pin Hsu ${ }^{3,4,8} \bowtie$

Scaphoid fractures are the most common carpal fractures. Diagnosing scaphoid fractures is challenging. Recently, cone-beam computed tomography (CBCT) has been shown to be a promising strategy for diagnosing scaphoid fractures. The diagnostic performance of $C B C T$ remains inconclusive in the literature. Through a systematic review and meta-analysis, our study aims to determine the diagnostic performance of $\mathrm{CBCT}$ for diagnosing scaphoid fractures. Five databases were searched up to March 25, 2020. We included prospective and retrospective studies describing the diagnostic accuracy of CBCT for scaphoid fractures in adult patients. QUADAS- 2 tool was used to assess the quality of the included studies. Four studies $(n=350)$ were included in the meta-analysis. Three of the four studies had high bias risk. The result showed that CBCT had a pooled sensitivity of 0.88 and a pooled specificity of 0.99 for scaphoid fracture diagnosis. The heterogeneities of sensitivity and specificity were substantial. The area under the summary receiver operating characteristic curve was 0.98 . No significant publication bias was observed. The result suggested that the diagnostic performance of CBCT for scaphoid fracture was excellent. The certainty of current evidence is low. Further well-designed studies with large sample sizes are warranted to confirm this finding.

Scaphoid fractures are the most common carpal fractures, accounting for $60 \%-70 \%$ of all carpal fractures ${ }^{1}$. The reported incidence is approximately 10.6-29 per 100,000 people per year, with peak occurrence in men between the ages of 20 and 24 years ${ }^{2}$. Among patients with suspected scaphoid fractures, the prevalence of true fractures is estimated to be $5 \%-20 \%{ }^{3}$. Early scaphoid fracture diagnosis is crucial because scaphoid fractures have a high risk of long-term complication, such as nonunion, avascular necrosis, and carpal instability, without timely diagnosis and adequate treatment ${ }^{4}$.

Scaphoid fracture diagnosis has several challenges. Plain radiography is usually used as the first imaging tool; however, its diagnostic accuracy for scaphoid fracture is notoriously poor, with up to $25 \%$ unrecognized scaphoid fractures ${ }^{5}$. When radiography shows a negative result or is inconclusive regarding fracture detection, additional cross-sectional imaging can be used to diagnose scaphoid fractures, including ultrasonography (US), computed tomography (CT), magnetic resonance imaging (MRI), and bone scintigraphy (BS). US is more accurate than plain radiography, with a reported pooled sensitivity of $85.6 \%$ and specificity of $83.3 \%{ }^{6}$. However, US cannot provide clear information on the fracture, such as the extent of dislocation and angulation, which may affect treatment decisions. Moreover, its operator-dependent diagnostic performance is limited ${ }^{7}$. CT is widely used and is more accurate than radiography and US, but it involves considerable radiation exposure ${ }^{8}$. MRI is

${ }^{1}$ Department of Emergency Medicine, Taoyuan Armed Forces General Hospital, Taoyuan, Taiwan. ${ }^{2}$ National Defense Medical Center, Taipei, Taiwan. ${ }^{3}$ Emergency Department, Wan Fang Hospital, Taipei Medical University, Taipei, Taiwan. ${ }^{4}$ Department of Emergency, School of Medicine, College of Medicine, Taipei Medical University, Taipei, Taiwan. ${ }^{5}$ Department of Orthopedic Surgery, Taoyuan Armed Forces General Hospital, Taoyuan, Taiwan. ${ }^{6}$ Department of Public Health, School of Medicine, College of Medicine, Taipei Medical University, Taipei, Taiwan. ${ }^{7}$ Department of Radiology, Tri-Service General Hospital, National Defense Medical Center, Taipei, Taiwan. ${ }^{8}$ Research Center of Big Data and Meta-Analysis, Wan Fang Hospital, Taipei Medical University, Taipei, Taiwan. ${ }^{\boxplus e m a i l}$ koakoahsu@gmail.com 
currently regarded as the reference standard in the literature, but it has a possibility of false positive results and cannot be obtained immediately in most institutions ${ }^{9}$. In a Cochrane meta-analysis, BS showed significantly higher diagnostic accuracy than $\mathrm{CT}$ and $\mathrm{MRI}^{10}$. However, it is more invasive than other modalities, has safety issues due to high radiation exposure, and has a diagnostic delay of at least $72 \mathrm{~h}$. Therefore, readily available and reliable imaging techniques for scaphoid fracture diagnosis are urgently needed.

Cone-beam computed tomography (CBCT) has been being used in oral and maxillofacial fields for $>20$ years, and it provides favorable visualization of details of small bony structures ${ }^{11}$. Compared with traditional or multidetector CT, it requires less space and provides higher spatial resolution under a possibly lower radiation dose exposure. Moreover, it is less invasive and more clinically accessible than MRI and BS. Therefore, using CBCT to diagnose scaphoid fractures is a promising strategy.

Recently, several studies have investigated the use of CBCT for diagnosing scaphoid fractures ${ }^{12-16}$. In these studies, diagnostic performance results have been inconsistent and have not been previously synthesized through a meta-analysis. We thus performed a systematic review and meta-analysis of the diagnostic performance of CBCT for scaphoid fractures.

\section{Method}

We registered our systematic review and meta-analysis protocol on PROSPERO (PROSPERO ID: CRD42020176017). Our study adhered to the Preferred Reporting Items for Systematic Reviews and MetaAnalyses statement ${ }^{17}$. Ethical approval or patient consent was not required as the present study was a review of previously published articles.

We systematically searched PubMed, Embase, Cochrane Library, Web of Science, and Scopus from inception up to March 25, 2020. Search keywords included fracture, scaphoid, wrist, and cone-beam computed tomography. Details of the search are provided in Supplementary Table 1. We included prospective and retrospective studies describing the diagnostic accuracy of CBCT for wrist bone fractures in adult patients (age $\geq 15$ years). Reviews, case series, case reports, conference proceedings, and animal studies were excluded. Moreover, studies focusing on soft tissue injury were excluded. No language restriction was applied. Two reviewers (TWY and YYL) screened all titles and abstracts to identify potentially eligible studies independently. The full text of potentially suitable articles was retrieved, and these articles were checked for inclusion by the two reviewers (TWY and YYL) independently. In case of disagreement, a third reviewer (YPH) made the final decision. We conducted the study selection procedure in EndNote, version 17 (Thomson Research Soft, Stamford, CT, USA). Finally, we examined the reference lists of all included studies to identify additional relevant studies.

Two investigators (KCWC and SCH) independently extracted data from the included studies. The following characteristics from each selected study were extracted: first author, publication year, study design, country, inclusion and exclusion criteria, sample size, age, sex, index test, reference standard, and the number of true positive, false positive, false negative, and true negative cases.

Two researchers (TWY and YYL) independently used Quality Assessment of Studies of Diagnostic Accuracy-2 to assess the quality of the included studies ${ }^{18}$. The tool has four domains, namely patient selection, index test, reference standard, and flow and timing. We assessed all domains for risk of bias and the first three domains for applicability concerns. We rated each domain as low risk, unclear risk, and high risk. We summarized the result using Review Manager Version 5.3 (The Nordic Cochrane Centre, The Cochrane Collaboration, Copenhagen, Denmark). Disagreements were resolved through discussion.

We used a bivariate random-effects model to calculate diagnostic test accuracy variables, including sensitivity, specificity, positive likelihood ratio (PLR), negative likelihood ratio (NLR), and diagnostic odds ratio. The area under the summary receiver operating characteristic (SROC) curve was calculated. All data were calculated with 95\% confidence intervals (CIs). We assessed the heterogeneity using the chi-square test and $\mathrm{I}^{2}$ statistics. $\mathrm{P}<0.1$ or $\mathrm{I}^{2}>50 \%$ suggested substantial heterogeneity. The publication bias in meta-analyses of diagnostic test accuracy was assessed using Deek's funnel plot asymmetry tes $\mathrm{t}^{19}$. We completed the meta-analysis with the MIDAS module for StataMP, version 14 (StataCorp LP, College Station, Texas).

We used Bayes theorem to estimate the posttest probability of scaphoid fracture. The posttest probability was calculated by multiplying pretest odds with the likelihood ratio. The pretest odds are calculated by dividing the pretest probability by (1-pretest probability). On the basis of pooled PLR and NLR, we used Fagan plot analysis to estimate posttest probabilities with the pretest probabilities of $25 \%, 50 \%$, and $75 \%$, respectively.

\section{Results}

Study selection. The flow diagram of study selection is displayed in Fig. 1. In total, 127 citations were identified after searching relevant databases. After removing duplicate records and excluding irrelevant studies through screening of titles and abstracts, 15 studies were selected in the full-text review stage. Of these, 11 were excluded because they comprised of reviews $(n=2)$, a case report $(n=1)$, did not involve the target population $(n=3)$ and did not examine the outcome of interest $(n=5)$. Finally, four ${ }^{12-15}$ studies met the inclusion criteria.

Study characteristics. The characteristics of the studies included in the meta-analysis are summarized in Table 1. All of the four ${ }^{12-15}$ studies were performed in Europe. Three ${ }^{12,13,15}$ were prospective studies, and one $^{14}$ was a retrospective study. The participants mainly included patients aged $\geq 15$ years. The mean age of the included participants was between 33 and 41 years. Three ${ }^{12-14}$ studies included patients with clinically suspected scaphoid fractures, and one ${ }^{15}$ study included patients with suspected radiocarpal fractures. The sample sizes ranged from 49 to 117 . All studies ${ }^{12-15}$ included more men than women and used CBCT as the index test. The details of the CBCT technique are summarized in Table 2. All studies ${ }^{12-15}$ included MRI as a reference standard; three $e^{12,14,15}$ of the studies used multiple reference standards through combination with other imaging modalities. 

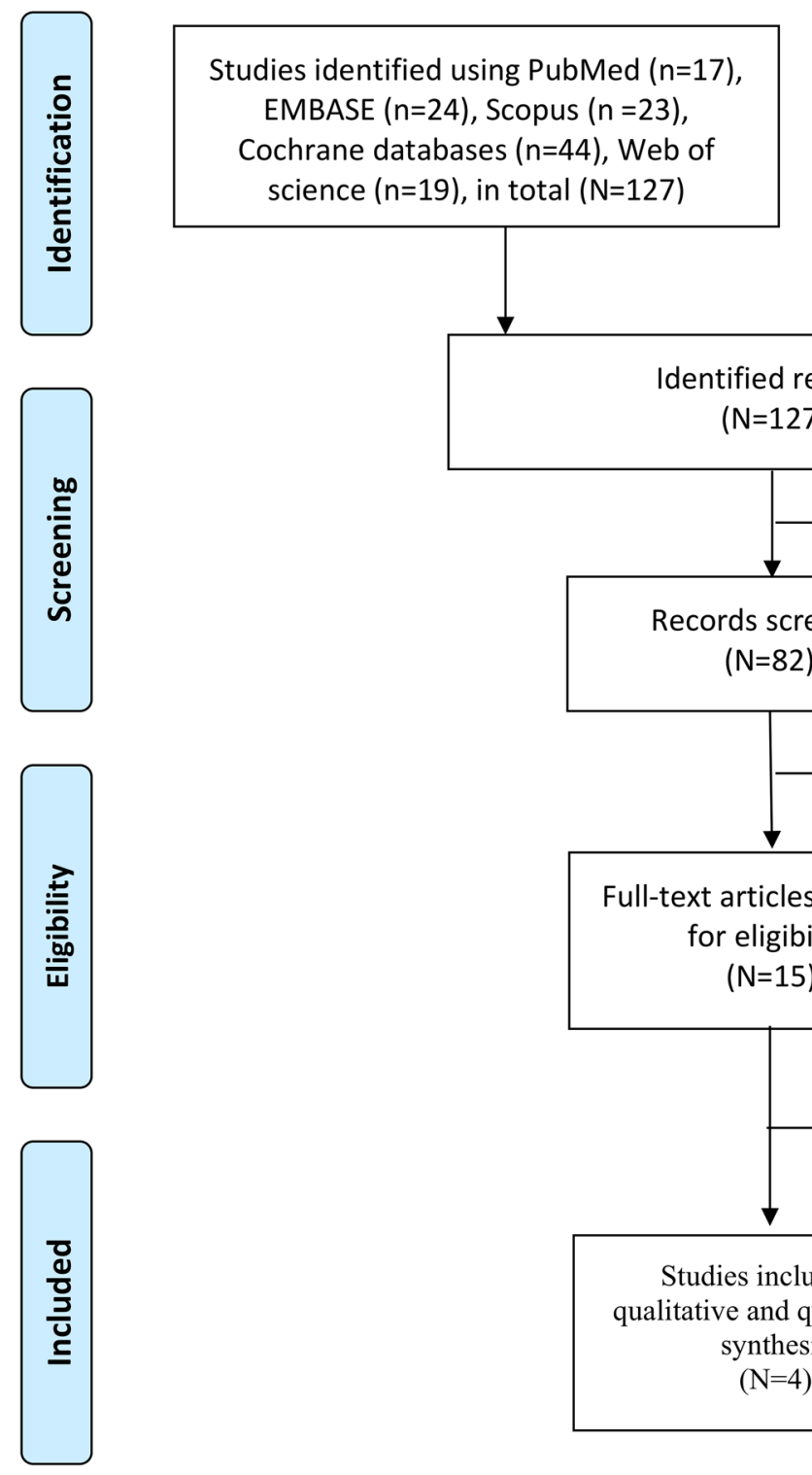

Additional studies identified by hand searching of the references of relevant

papers $(n=0)$

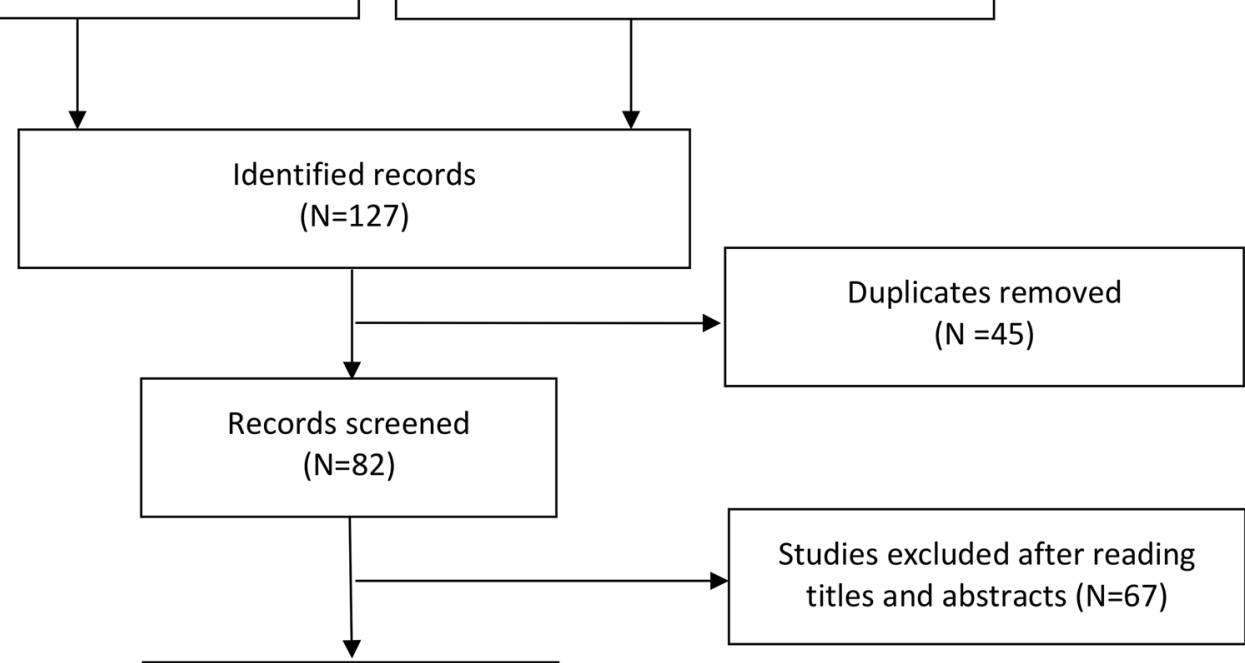

Full-text articles assessed for eligibility

$(\mathrm{N}=15)$

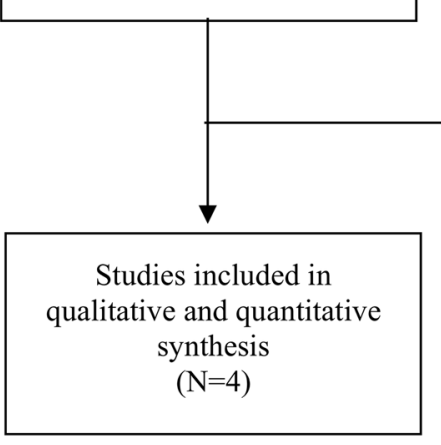

Full-text articles excluded, with reasons :( $N=11)$

Review ( $n=2)$

Case report $(n=1)$

Not target population $(n=3)$

Not outcome of interest $(n=5)$

Figure 1. Flowchart of study selection for the current meta-analysis.

\begin{tabular}{|c|c|c|c|c|c|c|c|c|}
\hline Study & Country & Design & Inclusion criteria & Sample size & Age, year (mean) & $\operatorname{Sex}(F / M)$ & Index test & Reference standard \\
\hline Gibney $^{15}$ & Ireland & Prospective & $\begin{array}{l}\text { Suspected radiocarpal fracture } \\
\text { but had normal radiographs; } \\
\text { age } \geq 16 \text { years; CBCT was per- } \\
\text { formed within } 14 \text { days }\end{array}$ & 117 & 41 & $57 / 60$ & СВСТ & $\begin{array}{l}\text { Consensus of two radiologists } \\
\text { regarding radiography, CBCT, and } \\
\text { MRI results }\end{array}$ \\
\hline Neubauer $^{14}$ & Germany & Retrospective & $\begin{array}{l}\text { Suspected scaphoid fracture and } \\
\text { had CBCT; age }>18 \text { years }\end{array}$ & 102 & 33 & $20 / 82$ & СВСТ & $\begin{array}{l}\text { Consensus of two radiologists } \\
\text { regarding radiography, CBCT, MRI } \\
\text { results }\end{array}$ \\
\hline Borel $^{13}$ & France & Prospective & $\begin{array}{l}\text { Suspected scaphoid fracture } \\
\text { but had normal radiographs; } \\
\text { age }>18 \text { years }\end{array}$ & 49 & 36 & $18 / 31$ & СВСТ & MRI \\
\hline Edlund $^{12}$ & Sweden & Prospective & $\begin{array}{l}\text { Clinical suspicion of scaphoid } \\
\text { fracture; age } \geq 15 \text { years }\end{array}$ & 95 & 40 & $38 / 57$ & СBCT & CBCT, MRI \\
\hline
\end{tabular}

Table 1. Characteristics of included studies. 


\begin{tabular}{|l|l|l|l|l|l|l|l|}
\hline Study & $\begin{array}{l}\text { CBCT scanner } \\
\text { (company) }\end{array}$ & Voltage/current & $\begin{array}{l}\text { Slice thickness/ } \\
\text { increment }\end{array}$ & Field of view & Matrix & $\begin{array}{l}\text { Acquisition } \\
\text { time }\end{array}$ & $\begin{array}{l}\text { Processing } \\
\text { time }\end{array}$ \\
\hline Gibney $^{15}$ & $\begin{array}{l}\text { Planmed Verity } \\
\text { (Planmed) }\end{array}$ & $90 \mathrm{kV} / 6 \mathrm{~mA}$ & $0.2 \mathrm{~mm} / \mathrm{NA}$ & $150 \times 150 \mathrm{~mm}$ & $\mathrm{NA}$ & $30 \mathrm{~s}$ & $1 \mathrm{~min}$ \\
\hline Neubauer $^{14}$ & $\begin{array}{l}\text { Planmed Verity } \\
\text { (Planmed) }\end{array}$ & $90 \mathrm{kV} / 36 \mathrm{~mA}$ & $0.2 \mathrm{~mm} / \mathrm{NA}$ & $\mathrm{NA}$ & $801 \times 801$ & $\mathrm{NA}$ & $\mathrm{NA}$ \\
\hline Borel $^{13}$ & $\begin{array}{l}\text { ProMax 3D mid } \\
\text { (Planmeca) }\end{array}$ & $90 \mathrm{kV} / 120 \mathrm{~mA}$ & $0.5 \mathrm{~mm} / \mathrm{NA}$ & $90 \times 90 \mathrm{~mm}$ & $\mathrm{NA}$ & $15 \mathrm{~s}$ & $\mathrm{NA}$ \\
\hline Edlund $^{12}$ & $\begin{array}{l}\text { Planmed Verity } \\
\text { (Planmed) }\end{array}$ & $\begin{array}{l}80-96 \mathrm{kV} / 6- \\
12 \mathrm{~mA}\end{array}$ & $\begin{array}{l}0.2-1 \mathrm{~mm} / 0.4- \\
0.5 \mathrm{~mm}\end{array}$ & $130 \times 160 \mathrm{~mm}$ & $\mathrm{NA}$ & $36 \mathrm{~s}$ & $1 \mathrm{~min}$ \\
\hline
\end{tabular}

Table 2. Details of the CBCT protocol. NA not available.

A

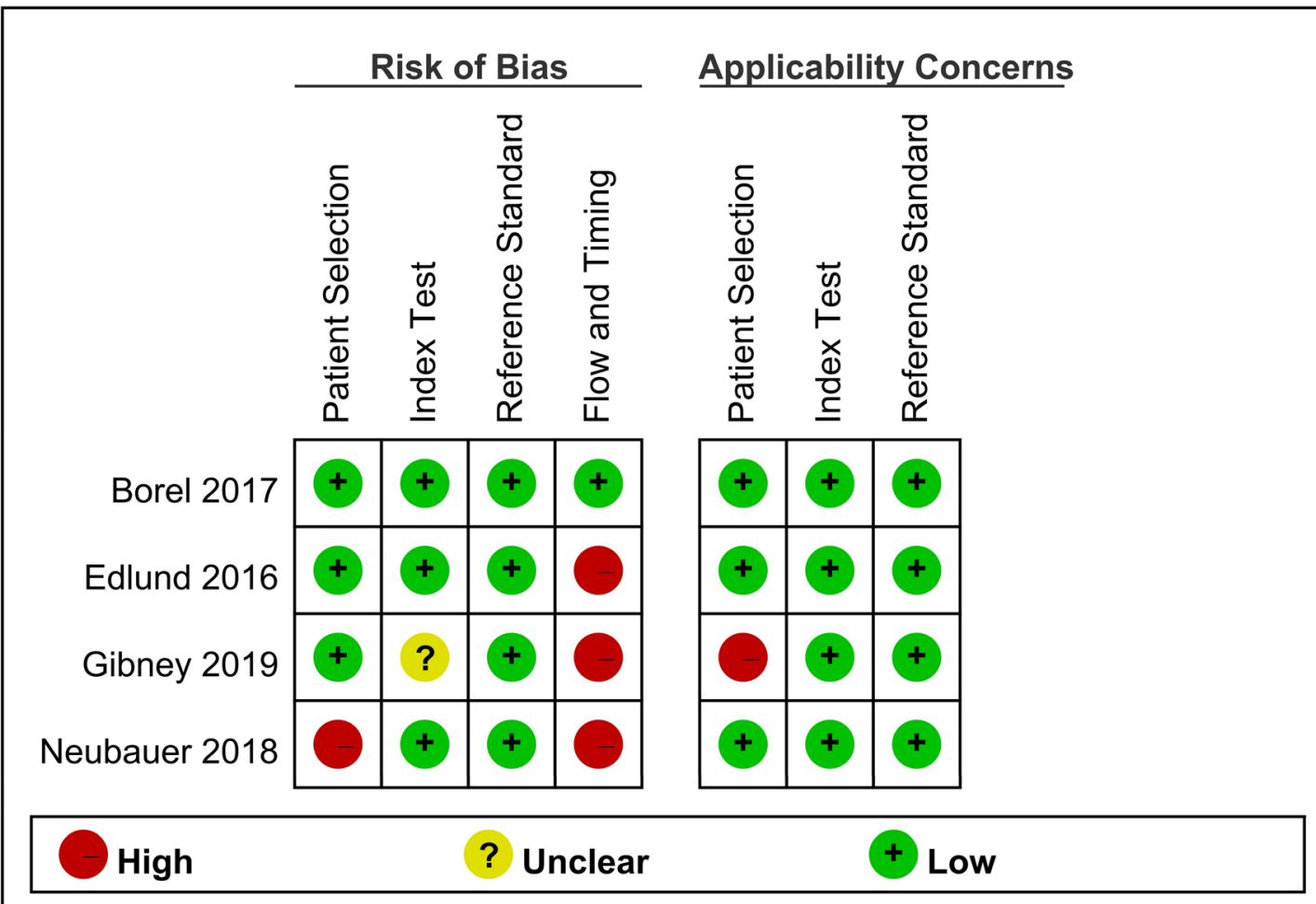

B

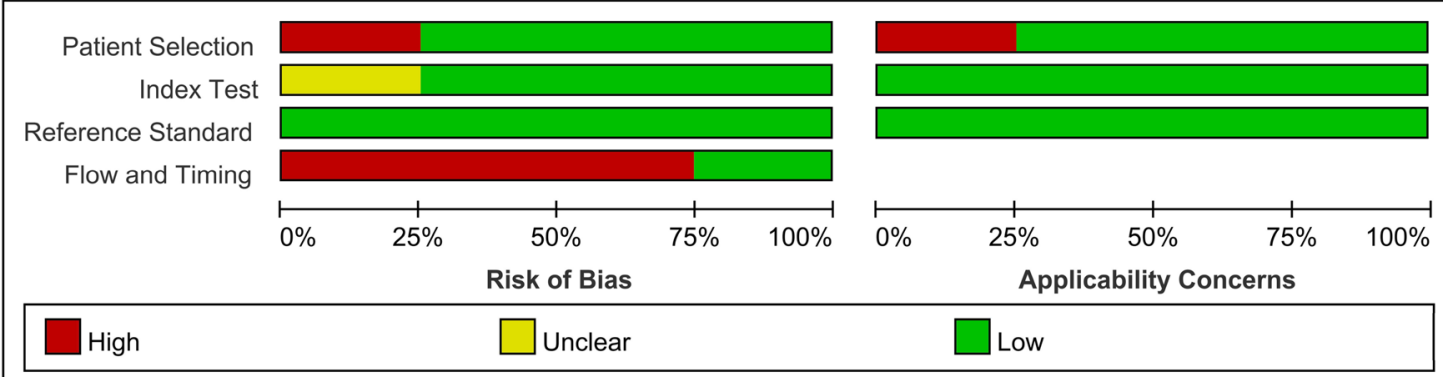

Figure 2. Methodological quality of included studies. 


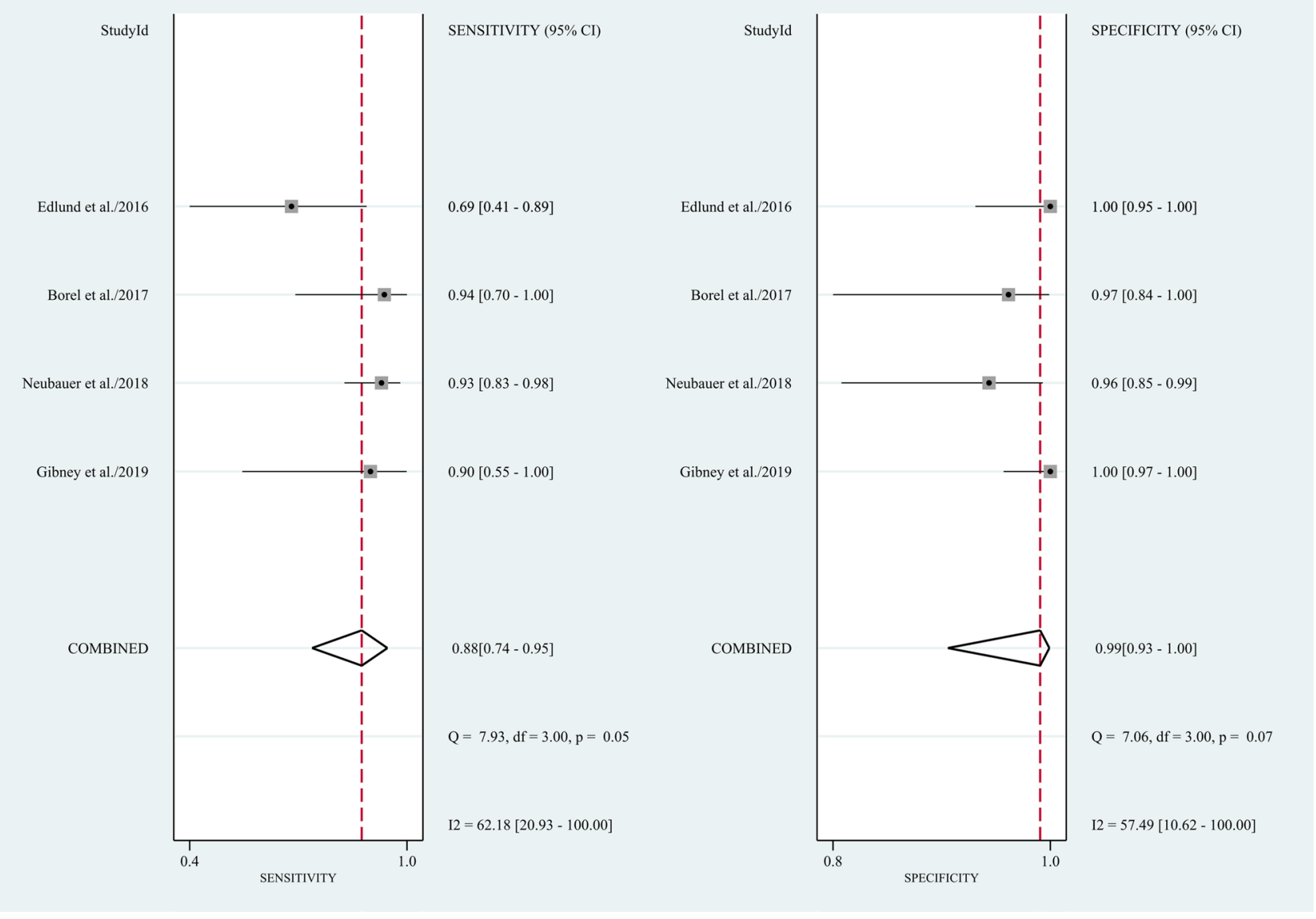

Figure 3. Forest plots of sensitivity and specificity for CBCT in scaphoid fracture diagnosis. Point estimates of sensitivity and specificity from each study are shown as solid circles. Error bars represented as 95\% CIs.

Bias risk and applicability of the included studies. Figure 2 presents the methodological quality assessment of bias risk of eligible studies. One ${ }^{13}$ study was rated as low bias risk and low concern of patient applicability. Three studies ${ }^{12,14,15}$ were rated as high bias risk because, in one ${ }^{14}$ of the three studies, the authors did not consecutively recruit patients suspected of having scaphoid fractures, and not all patients in these three ${ }^{12,14,15}$ studies received the same reference standard. Concerning applicability, one ${ }^{15}$ study used a retrospective design with high concerns of patient applicability.

Meta-analysis of CBCT for diagnosing scaphoid fractures. Four ${ }^{12-15}$ studies $(\mathrm{n}=350)$ were included in the meta-analysis. The result indicated that CBCT had a pooled sensitivity of 0.88 (95\% CI $0.74-0.95$, $\mathrm{I}^{2}=62.18$; Fig. 3) and a pooled specificity of $0.99\left(95 \%\right.$ CI $0.93-1.00, \mathrm{I}^{2}=57.49$; Fig. 3) for scaphoid fracture diagnosis. Notably, heterogeneities among studies were substantial. This resulted in a PLR of 119.0 (95\% CI 11.7-1210.2; Supplementary Table 2), NLR of 0.12 (95\% CI 0.06-0.27; Supplementary Table 2), and Diagnostic odds ratio (DOR) of 957 (95\% CI 110-8346; Supplementary Table 2). The area under the SROC curve indicated a high accuracy of CBCT in scaphoid fracture diagnosis (0.98, 95\% CI 0.96-0.99; Fig. 4). Funnel plot analysis indicated no significant publication bias ( $p=0.27$; Fig. 5).

\section{Discussion}

To the best of our knowledge, this is the first systematic review and meta-analysis to investigate the diagnostic performance of CBCT for scaphoid fractures. The result of our meta-analysis demonstrated that CBCT has both high sensitivity and high specificity for scaphoid fracture diagnosis.

Scaphoid fracture diagnosis remains challenging for clinicians. Currently, for scaphoid fracture diagnosis, clinical history, physical examination, plain radiography, and further imaging are included. Clinical history ${ }^{10}$, physical examinations ${ }^{10}$, and plain radiography ${ }^{20}$ have insufficient sensitivity and specificity for scaphoid fracture diagnosis. Further cross-sectional imaging is advocated for susceptible patients. A guideline in the United Kingdom suggests BS, CT, and MRI for suspected fractures ${ }^{21}$. Furthermore, another guideline in the United States suggests $\mathrm{MRI}^{22}$. A recent meta-analysis showed that MRI has the highest sensitivity and specificity for detecting scaphoid fractures ${ }^{8}$. However, CT is widely used in current practice because it is easily available and costs less than MRI ${ }^{8}$. Mallee et al. showed that CT has a sensitivity of $72 \%$ and specificity of $99 \%$ for diagnosing scaphoid 


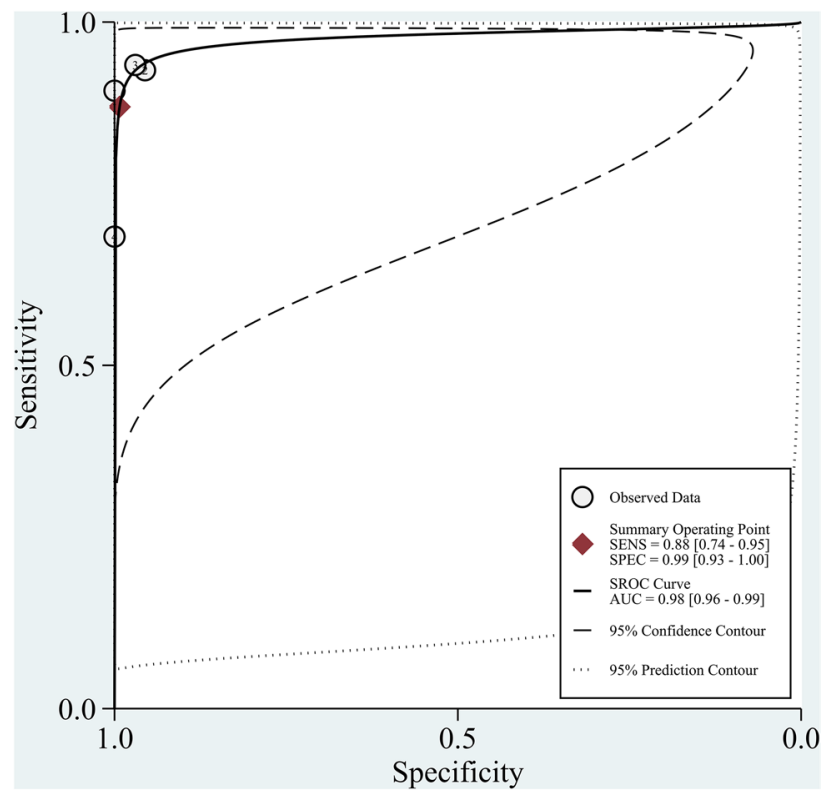

Figure 4. SROC curve for CBCT in scaphoid fracture detection.

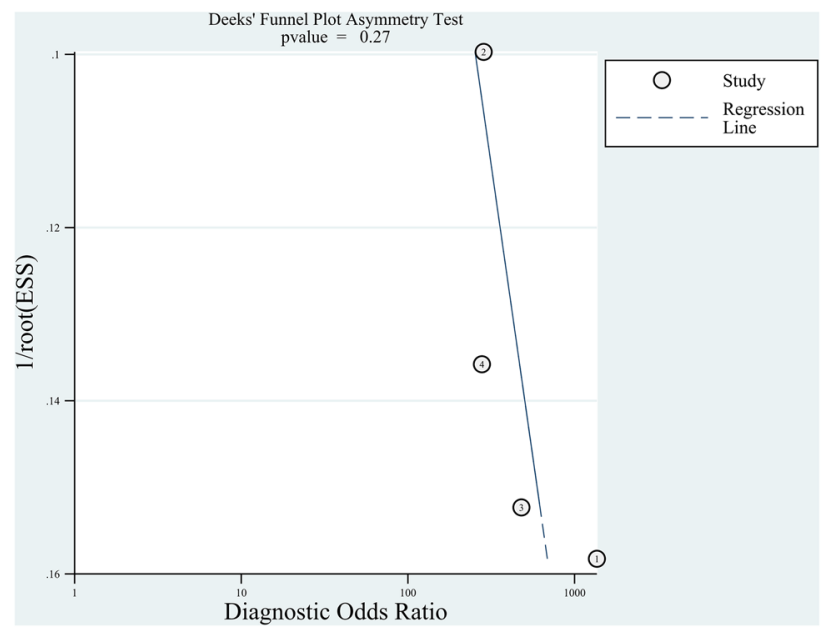

Figure 5. Deek's funnel plot asymmetry test for publication bias for CBCT.

fractures ${ }^{10}$. In our study, CBCT had a specificity of $99 \%$ and sensitivity of $88 \%$ for diagnosing scaphoid fractures, which implies that CBCT has higher diagnostic performance than CT.

Recent studies on $\mathrm{CBCT}$ have shown that $\mathrm{CBCT}$ may replace conventional CT for scaphoid fracture diagnosis $^{23,24}$. Practically, CBCT requires a relatively small space, which makes it easier for medical institutions to retrofit the new space. One of the main differences between CBCT and CT is that CBCT has a higher spatial resolution ${ }^{25-27}$, which makes it a more effective tool for bone visualization ${ }^{24}$. Moreover, CBCT is associated with a lower radiation dose than CT without optimizing the examination protocols ${ }^{28-30}$. Tschauner et al. found that adapted extremity CBCT imaging protocols can fall below optimized pediatric ankle and wrist CT doses at comparable image qualities ${ }^{31}$. Additionally, CBCT showed excellent agreement with CT to confirm fractures in patients with distal limb trauma ${ }^{32}$. Moreover, two studies have demonstrated that CBCT has high interrater agreement with CT for scaphoid fracture diagnosis ${ }^{13,15}$. Furthermore, Honigmann et al. indicated that CBCT is more cost-effective than CT and MRI ${ }^{33}$.

Despite these aforementioned prospects, some disadvantages may limit CBCT application. The acquisition time required by CBCT makes it prone to motion artifact ${ }^{34}$. However, Spin-Neto et al. observed that the automated motion artifact-correction system significantly enhanced CBCT image quality and interpretability ${ }^{35}$. Regarding soft tissue visualization, CBCT performs worse than $\mathrm{CT}^{24}$. Moreover, metallic implants produce beam hardening artifacts in CBCT, and sufficient evidence is lacking for CBCT use in the postoperative evaluation of patients with implants ${ }^{36}$. Moreover, CT is diagnostically accurate for scaphoid fracture despite being slightly inferior to $\mathrm{CBCT}^{10,13}$. Furthermore, $\mathrm{CT}$ shows high diagnostic accuracy for trauma, brain, chest, and abdominal 
emergency and has wide clinical application ${ }^{37,38}$. Consequently, despite giving a detailed visualization of small bony structures, CBCT only provides a small field of view, which may limit its widespread use. Therefore, in clinical practice, at medical institutions equipped with CBCT, clinicians may consider using CBCT instead of CT for the diagnosis of patients suspected with scaphoid fracture.

Importantly, the pretest probability of the disease may influence the application of CBCT for scaphoid fracture. When we applied a pretest probability of $25 \%$, the posttest probabilities of positive and negative CBCTs were $98 \%$ and $4 \%$, respectively; when we applied a pretest probability of $50 \%$, the posttest probabilities of positive and negative CBCTs were $99 \%$ and $11 \%$, respectively. When we applied a pretest probability of $75 \%$, the posttest probabilities of positive and negative CBCTs were $99.7 \%$ and $27 \%$, respectively (Supplementary Fig. 1). Clinicians could reliably confirm the disease through CBCT with evidence of scaphoid fracture. However, for a negative examination, clinicians had insufficient information to exclude the diagnosis when the scaphoid fracture prevalence was $>50 \%$. In the included studies, the overall prevalence ranged from $8.6 \%$ to $52.0 \%$. Two ${ }^{13,14}$ of the included studies in our meta-analysis had a prevalence rate of $>50 \%$, which is higher than that reported $5 \%-20 \%$ in the literature ${ }^{3}$. Thus, different studies included patients with different demographic and clinical factors for the prediction of a true fracture. Duckworth et al. indicated that the predictors of a true scaphoid fracture included male gender, sports injury, anatomical snuffbox pain on the ulnar deviation of the wrist, and pain on thumb-index finger pinch at presentation; predictors at the 2-week review were scaphoid tubercle tenderness ${ }^{39}$. These predictors raised the prevalence of true fractures among suspected fractures. Subsequently, they improved the diagnostic accuracy and lowered the impact on clinical practice. Taken together, the results indicate that in these high-risk patients with a negative CBCT result, a scaphoid fracture cannot be excluded and needs immobilization in a splint.

The timing of testing may have an essential role in the diagnostic accuracy of imaging for scaphoid fracture. Among the included studies in our meta-analysis ${ }^{12-15}$, the timing for applying CBCT varied after the inciting event. CBCT was performed on the first day in Edlund's study ${ }^{12}$, within 7 days in Borel's study ${ }^{13}$, within 4 days in Neubauer's study ${ }^{14}$, and within 7-14 days in Gibney's study ${ }^{15}$. Nonetheless, Kumar et al. found that the sensitivity of MRI within $24 \mathrm{~h}$ after trauma was comparable to day 10 after initial presentation ${ }^{40}$. Moreover, the early use of MRI resulted in low pain and high satisfaction scores as well as less time for immobilization, early treatment, and less time off work and did not increase health $\operatorname{costs}^{41}$. Another study indicated that early CT or MRI significantly reduced diagnosis time ${ }^{42}$. Given these potential social benefits, we suggest early CBCT, from 1 to 14 days of injury, to be the management technique of choice. Further studies are needed to clarify the optimal timing for CBCT.

Our study has several limitations. First, the results were based on a limited number of included observational studies with relatively small sample sizes. The precision for summary estimates may be insufficient. Second, three of four included studies were rated as high risk of bias due to the use of multiple reference standards. Third, we found substantial heterogeneities of pooled sensitivity and specificity, which may be attributed to different clinical manifestations of the included participant, different study design, various protocols of CBCT, and the use of different reference standards among studies. Fourth, although we did not detect publication bias, the finding was not powerful because $<10$ studies were included. The aforementioned limitations may downgrade the certainty of evidence. Moreover, all studies were conducted in Europe. Therefore, further investigation is required before generalizing these findings to other continents.

In conclusion, the literature suggests that $\mathrm{CBCT}$ is both sensitive and specific for scaphoid fracture diagnosis. CBCT exhibits higher diagnostic performance than CT and should be considered as a replacement when the institution is equipped with CBCT. For high-risk patients, a negative result of CBCT should be interpreted with caution. Further studies are needed before routine implementation because of the current low certainty of evidence.

\section{Data availability}

All data generated or analyzed during this systematic review are included in this published article (and its Supplementary File).

Received: 8 June 2020; Accepted: 19 January 2021

Published online: 28 January 2021

\section{References}

1. Hove, L. M. Epidemiology of scaphoid fractures in Bergen, Norway. Scand. J. Plast. Reconstr. Surg. Hand Surg. 33, 423-426 (1999).

2. Garala, K., Taub, N. A. \& Dias, J. J. The epidemiology of fractures of the scaphoid: Impact of age, gender, deprivation and seasonality. Bone Joint J. 98, 654-659 (2016).

3. Buijze, G. \& Jupiter, J. B. Scaphoid Fractures: Evidence-based Management (Elsevier Health Sciences, Amsterdam, 2017).

4. Hackney, L. A. \& Dodds, S. D. Assessment of scaphoid fracture healing. Curr. Rev. Musculoskelet. Med. 4, 16-22 (2011).

5. Jenkins, P. J., Slade, K., Huntley, J. S. \& Robinson, C. M. A comparative analysis of the accuracy, diagnostic uncertainty and cost of imaging modalities in suspected scaphoid fractures. Injury 39, 768-774 (2008).

6. Kwee, R. M. \& Kwee, T. C. Ultrasound for diagnosing radiographically occult scaphoid fracture. Skeletal Radiol. 47, 1205-1212 (2018).

7. Ali, M., Ali, M., Mohamed, A., Mannan, S. \& Fallahi, F. The role of ultrasonography in the diagnosis of occult scaphoid fractures. J. Ultrason. 18, 325-331 (2018).

8. Backer, H. C., Wu, C. H. \& Strauch, R. J. Systematic review of diagnosis of clinically suspected scaphoid fractures. J. Wrist Surg. 9, 81-89 (2020).

9. De Zwart, A. D. et al. MRI as a reference standard for suspected scaphoid fractures. Br. J. Radiol. 85, 1098-1101 (2012).

10. Mallee, W. H. et al. Computed tomography versus magnetic resonance imaging versus bone scintigraphy for clinically suspected scaphoid fractures in patients with negative plain radiographs. Cochrane Database Syst. Rev. 1, 010023 (2015). 
11. Loubele, M., Guerrero, M. E., Jacobs, R., Suetens, P. \& van Steenberghe, D. A comparison of jaw dimensional and quality assessments of bone characteristics with cone-beam CT, spiral tomography, and multi-slice spiral CT. Int. J. Oral Maxillofac Implants 22, 446-454 (2007).

12. Edlund, R., Skorpil, M., Lapidus, G. \& Backlund, J. Cone-Beam CT in diagnosis of scaphoid fractures. Skeletal Radiol. 45, 197-204 (2016).

13. Borel, C. et al. Diagnostic value of cone beam computed tomography (CBCT) in occult scaphoid and wrist fractures. Eur. J. Radiol. 97, 59-64 (2017).

14. Neubauer, J. et al. Comparison of the diagnostic accuracy of cone beam computed tomography and radiography for scaphoid fractures. Sci. Rep. 8, 3906 (2018).

15. Gibney, B. et al. Incorporating cone-beam CT into the diagnostic algorithm for suspected radiocarpal fractures: A new standard of care?. Am. J. Roentgenol. 213, 1117-1123 (2019).

16. Ricci, P. M. et al. Cone-beam computed tomography compared to X-ray in diagnosis of extremities bone fractures: A study of 198 cases. Eur. J. Radiol. Open 6, 119-121 (2019).

17. Moher, D., Liberati, A., Tetzlaff, J. \& Altman, D. G. Preferred reporting items for systematic reviews and meta-analyses: The PRISMA statement. PLoS Med. 6, e1000097 (2009).

18. Whiting, P. F. et al. QUADAS-2: A revised tool for the quality assessment of diagnostic accuracy studies. Ann. Intern. Med. 155, 529-536 (2011).

19. Deeks, J. J., Macaskill, P. \& Irwig, L. The performance of tests of publication bias and other sample size effects in systematic reviews of diagnostic test accuracy was assessed. J. Clin. Epidemiol. 58, 882-893 (2005).

20. Carpenter, C. R. et al. Adult scaphoid fracture. Acad. Emerg. Med. 21, 101-121 (2014).

21. Party, R. W. Making the Best Use of a Department of Clinical Radiology: Guidelines for Doctors (Royal College of Radiologists, London, 2003).

22. Radiology, A. C. O. Expert Panel on Musculoskeletal Imaging ACR Appropriateness Criteria 1-7 (American College of Radiology, Philadelphia, 2001).

23. Neubauer, J. et al. Comparing the image quality of a mobile flat-panel computed tomography and a multidetector computed tomography: A phantom study. Invest. Radiol. 49, 491-497 (2014).

24. Demehri, S. et al. Assessment of image quality in soft tissue and bone visualization tasks for a dedicated extremity cone-beam CT system. Eur. Radiol. 25, 1742-1751 (2015).

25. Lechuga, L. \& Weidlich, G. A. Cone beam CT vs. fan beam CT: A comparison of image quality and dose delivered between two differing CT imaging modalities. Cureus 8, e778 (2016).

26. Koskinen, S. K. et al. CT arthrography of the wrist using a novel, mobile, dedicated extremity cone-beam CT (CBCT). Skeletal Radiol. 42, 649-657 (2013).

27. Watanabe, H., Honda, E., Tetsumura, A. \& Kurabayashi, T. A comparative study for spatial resolution and subjective image characteristics of a multi-slice CT and a cone-beam CT for dental use. Eur. J. Radiol. 77, 397-402 (2011).

28. Ramdhian-Wihlm, R. et al. Cone-beam computed tomography arthrography: An innovative modality for the evaluation of wrist ligament and cartilage injuries. Skeletal Radiol. 41, 963-969 (2012).

29. Neubauer, J. et al. Comparison of the radiation dose from cone beam computed tomography and multidetector computed tomography in examinations of the hand. Rofo 188, 488-493 (2016).

30. Hofmann, E. et al. Comparative study of image quality and radiation dose of cone beam and low-dose multislice computed tomography-an in-vitro investigation. Clin. Oral Investig. 18, 301-311 (2014).

31. Tschauner, S. et al. Surface radiation dose comparison of a dedicated extremity cone beam computed tomography (CBCT) device and a multidetector computed tomography (MDCT) machine in pediatric ankle and wrist phantoms. PLoS ONE 12, e0178747 (2017).

32. Dubreuil, T. et al. Comparison of cone-beam computed tomography and multislice computed tomography in the assessment of extremity fractures. J. Comput. Assist. Tomogr. 43, 372-378 (2019).

33. Honigmann, P. \& Frueh, F. S. Letter about a published paper. J. Hand. Surg. Eur. 43, 451 (2018)

34. Posadzy, M., Desimpel, J. \& Vanhoenacker, F. Cone beam CT of the musculoskeletal system: Clinical applications. Insights Imaging 9, 35-45 (2018).

35. Spin-Neto, R., Matzen, L. H., Schropp, L. W., Sorensen, T. S. \& Wenzel, A. An ex vivo study of automated motion artefact correction and the impact on cone beam CT image quality and interpretability. Dentomaxillofac Radiol. 47, 20180013 (2018).

36. Pallaver, A. \& Honigmann, P. The role of cone-beam computed tomography (CBCT) scan for detection and follow-up of traumatic wrist pathologies. J. Hand Sur. Am. 44, 1081-1087 (2019).

37. Salimi, J. et al. Diagnostic accuracy of CT scan in abdominal blunt trauma. Chin. J. Traumatol. 12, 67-70 (2009).

38. Liguori, C. et al. Emerging clinical applications of computed tomography. Med. Devices 8, 265-278 (2015).

39. Duckworth, A. D. et al. Predictors of fracture following suspected injury to the scaphoid. J. Bone Joint Surg. Br. 94, 961-968 (2012).

40. Kumar, S., O'Connor, A., Despois, M. \& Galloway, H. Use of early magnetic resonance imaging in the diagnosis of occult scaphoid fractures: The CAST Study (Canberra Area Scaphoid Trial). N. Z. Med. J. 118, U1296 (2005).

41. Kelson, T., Davidson, R. \& Baker, T. Early MRI versus conventional management in the detection of occult scaphoid fractures: What does it really cost? A rural pilot study. J. Med. Radiat. Sci. 63, 9-16 (2016).

42. Wijetunga, A. R., Tsang, V. H. \& Giuffre, B. The utility of cross-sectional imaging in the management of suspected scaphoid fractures. J. Med. Radiat. Sci. 66, 30-37 (2019).

\section{Acknowledgements}

This work was supported by the project of Wan Fang Hospital, Taipei Medical University, Taipei, Taiwan (Grant No.: 109-wf-eva-19 to Yuan-Pin Hsu). The funders had no role in study design, data collection and analysis, decision to publish, or manuscript preparation.

\section{Author contributions}

T.-W.Y. and Y.-P.H. had full access to all the data in the study and take responsibility for the integrity of the data and the accuracy of the data analysis. Concept and design: Y.-P.H. Acquisition, analysis, and interpretation of data: T.-W.Y., Y.-Y.L., C.-W.H., C.-W.H., K.C.-W.C., S.-C.H., C.-H.B., C.-K.C., and Y.-P.H. Drafting the manuscript: T.-W.Y. and Y.-P.H. Statistical analysis: C.-W.H. and C.-H.B. Study supervision: Y.-P.H.

\section{Competing interests}

The authors declare no competing interests. 


\section{Additional information}

Supplementary Information The online version contains supplementary material available at https://doi. org/10.1038/s41598-021-82351-9.

Correspondence and requests for materials should be addressed to Y.-P.H.

Reprints and permissions information is available at www.nature.com/reprints.

Publisher's note Springer Nature remains neutral with regard to jurisdictional claims in published maps and institutional affiliations.

(c) (1) Open Access This article is licensed under a Creative Commons Attribution 4.0 International cc) License, which permits use, sharing, adaptation, distribution and reproduction in any medium or format, as long as you give appropriate credit to the original author(s) and the source, provide a link to the Creative Commons licence, and indicate if changes were made. The images or other third party material in this article are included in the article's Creative Commons licence, unless indicated otherwise in a credit line to the material. If material is not included in the article's Creative Commons licence and your intended use is not permitted by statutory regulation or exceeds the permitted use, you will need to obtain permission directly from the copyright holder. To view a copy of this licence, visit http://creativecommons.org/licenses/by/4.0/.

(C) The Author(s) 2021 